\title{
Irrational Rotation of the Circle and the Binary Odometer are Finitarily Orbit Equivalent
}

\author{
By
}

Mrinal Kanti RoychowdHURY*

\begin{abstract}
Two invertible dynamical systems $(X, \mathfrak{A}, \mu, T)$ and $(Y, \mathfrak{B}, \nu, S)$ where $X, Y$ are metrizable spaces and $T, S$ are homeomorphisms on $X$ and $Y$, are said to be finitarily orbit equivalent if there exists an invertible measure preserving mapping $\phi$ from a subset $M$ of $X$ of measure one to a subset of $Y$ of full measure such that

(i) $\left.\phi\right|_{M}$ is continuous in the relative topology on $M$ and $\left.\phi^{-1}\right|_{\phi(M)}$ is continuous in the relative topology on $\phi(M)$,

(ii) $\phi\left(\operatorname{Orb}_{T}(x)\right)=\operatorname{Orb}_{S} \phi(x)$ for $\mu$-a.e. $x \in X$.

In this article a finitary orbit equivalence mapping has been constructed between an irrational rotation of the circle and the binary odometer.
\end{abstract}

\section{$\S 1$. Introduction}

The theory of orbit equivalence originated in the late 1950's. H. Dye pioneered the early development of the theory in his article [D] "On groups of measure preserving transformations I". In this work he proved one of the most fundamental theorems in ergodic theory, that any two invertible ergodic measure preserving transformations acting on non-atomic Lebesgue probability spaces are orbit equivalent, i.e. there is a measure isomorphism between the spaces taking complete orbits to complete orbits, modulo the underlying probability measures. This result is usually [HO] proved by taking the binary

Communicated by Y. Takahashi. Received May 9, 2005. Revised January 25, 2006, May $16,2006$.

2000 Mathematics Subject Classification(s): 28D05, 37A05, 37A20.

Key words: finitary isomorphism, orbit equivalence, odometer and irrational rotation.

* This work was done in partial fulfillment of the author's Ph.D. at Wesleyan University under the direction of Prof Michael S. Keane.

Current address: Dept of Mathematics, Colorado State University, CO 80523, USA.

(c) 2007 Research Institute for Mathematical Sciences, Kyoto University. All rights reserved. 
odometer, whose ergodic structure is well understood, as one of the transformations.

There has been considerable interest in orbit equivalence theory since Dye's original article, and many other aspects of orbit equivalence have been studied. In particular the broad study of finitary orbit equivalence were begun by Hamachi and Keane [HK].

Two invertible dynamical systems $(X, \mathfrak{A}, \mu, T)$ and $(Y, \mathfrak{B}, \nu, S)$ where $X, Y$ are metrizable spaces and $T, S$ are homeomorphisms on $X$ and $Y$, are said to be finitarily orbit equivalent if there exists an invertible measure preserving mapping $\phi$ from a subset $M$ of $X$ of measure one to a subset of $Y$ of full measure such that

(i) $\left.\phi\right|_{M}$ is continuous in the relative topology on $M$ and $\left.\phi^{-1}\right|_{\phi(M)}$ is continuous in the relative topology on $\phi(M)$,

(ii) $\phi\left(\operatorname{Orb}_{T}(x)\right)=\operatorname{Orb}_{S} \phi(x)$ for $\mu$-a.e. $x \in X$.

It remains an open problem as to whether any two orbit equivalent systems are finitarily orbit equivalent. In 2003, in the article [HK] Hamachi and Keane constructed the first finitary orbit equivalence mapping between the binary odometer and the ternary odometer. In this article we prove the theorem:

Main Theorem. Irrational rotation of the circle and the binary odometer are finitarily orbit equivalent.

The finitary theory began in the study of isomorphism and this background can be found in [KS1], [KS2]. Background for the methods used in the finitary orbit equivalence theory are in $[\mathrm{HK}],[\mathrm{HKR}]$. Particularly note the result in $[\mathrm{R}]$ that any two odometers are finitarily orbit equivalent. This work is closest to what we do here.

The paper is arranged as follows. In Section 2 we discuss the theory of irrational rotation of the circle in order to define the basic notions of cylinders and tower partitions and establish for them the basic theorems from $[\mathrm{HK}],[\mathrm{R}]$. For completeness Section 3 does the same for the binary odometer. Section 4 discusses tower maps and Section 5 completes the finitary construction.

\section{§. Irrational Rotation of the Circle}

In this section we state the required definitions and some preliminary results concerning an irrational rotation of the circle. 
$\S$ 2.1. Irrational rotation of the circle, canonical cylinders, iterated cylinders

Let $\alpha \in(0,1)$ be irrational. Let $X=[0,1)=\mathbb{R} / \mathbb{Z}$. Then $X$ is endowed with the metric $d$, given by

$$
d(x, y)=\min \{|x-y|, 1-|x-y|\}
$$

for $x, y \in X$. This metric generates a topology on $X$. Let $\mathfrak{A}$ be the Borel $\sigma$-algebra generated by the topology on $X$ and $\mu$ be the Lebesgue measure on $(X, \mathfrak{A})$. Under the addition modulo $1, X$ is a compact topological group and $\mu$ is the normalized Haar measure on $X$. Moreover, since the subgroup generated by $\alpha$ is dense, $X$ is monothetic.

The invertible ergodic measure preserving transformation $T: X \rightarrow X$ given by $T x=x+\alpha(\bmod 1)$ is called the irrational rotation of the circle by $\alpha$. Note that $T$ is a homeomorphism, since it is the translation by the element $\alpha$ of the group.

Since $\alpha$ is an irrational number between 0 and 1 , we can write it in an infinite continued fraction expansion as

$$
\alpha=\frac{1}{a_{1}+\frac{1}{a_{2}+\frac{1}{a_{3}+\cdots}}}
$$

where $a_{1}, a_{2}, a_{3}, \cdots$ are positive integers. Let

$$
\begin{aligned}
\alpha_{0} & =\alpha \\
\alpha_{1} & =1-a_{1} \alpha_{0} \\
\alpha_{k+1} & =\alpha_{k-1}-a_{k+1} \alpha_{k} \quad(k \geq 1) .
\end{aligned}
$$

We now consider the two sequences $\left\{t_{1}^{(n)}: n \in \mathbb{N}\right\}$ and $\left\{t_{2}^{(n)}: n \in \mathbb{N}\right\}$ given by

$$
\left[\begin{array}{ll}
t_{1}^{(n)} & t_{2}^{(n)}
\end{array}\right]=\left[\begin{array}{ll}
a_{1} & 1
\end{array}\right]\left[\begin{array}{cc}
a_{2} & 1 \\
1 & 0
\end{array}\right]\left[\begin{array}{cc}
a_{3} & 1 \\
1 & 0
\end{array}\right] \ldots\left[\begin{array}{cc}
a_{n} & 1 \\
1 & 0
\end{array}\right] \quad(n \geq 1)
$$

For example if $n=1$,

$$
\left[\begin{array}{ll}
t_{1}^{(1)} & t_{2}^{(1)}
\end{array}\right]=\left[\begin{array}{ll}
a_{1} & 1
\end{array}\right]
$$

i.e. $t_{1}^{(1)}=a_{1}, t_{2}^{(1)}=1$.

If $n=2$,

$$
\left[\begin{array}{ll}
t_{1}^{(2)} & t_{2}^{(2)}
\end{array}\right]=\left[\begin{array}{ll}
a_{1} & 1
\end{array}\right]\left[\begin{array}{cc}
a_{2} & 1 \\
1 & 0
\end{array}\right]=\left[\begin{array}{ll}
a_{1} a_{2}+1 & a_{1}
\end{array}\right]
$$


i.e. $t_{1}^{(2)}=a_{1} a_{2}+1, t_{2}^{(2)}=a_{1}$ and so on for $n=3,4,5, \ldots$.

Note. That (1) implies:

$$
\left[\begin{array}{ll}
t_{1}^{(m+1)} & t_{2}^{(m+1)}
\end{array}\right]=\left[\begin{array}{ll}
t_{1}^{(m)} & t_{2}^{(m)}
\end{array}\right]\left[\begin{array}{cc}
a_{m+1} & 1 \\
1 & 0
\end{array}\right]=\left[\begin{array}{ll}
a_{m+1} t_{1}^{(m)}+t_{2}^{(m)} & t_{1}^{(m)}
\end{array}\right] .
$$

Hence

$$
t_{1}^{(m+1)}=a_{m+1} t_{1}^{(m)}+t_{2}^{(m)} \text { and } t_{2}^{(m+1)}=t_{1}^{(m)} \text { for } m \geq 1 .
$$

Let us write

$$
t^{(n)}=t_{1}^{(n)}+t_{2}^{(n)} \quad(n \geq 1)
$$

and consider the sets,

$$
\begin{aligned}
S_{0} & =\{0\} \\
S_{1} & =\left\{0, T 0, T^{2} 0, \ldots, T^{t^{(1)}-1} 0\right\} \\
S_{2} & =\left\{0, T 0, T^{2} 0, \ldots, T^{t^{(1)}-1} 0, T^{t^{(1)}} 0, \ldots, T^{t^{(2)}-1} 0\right\} \\
S_{3} & =\left\{0, T 0, T^{2} 0, \ldots, T^{t^{(1)}-1} 0, T^{t^{(1)}} 0, \ldots, T^{t^{(2)}-1} 0, T^{t^{(2)}} 0, \ldots, T^{t^{(3)}-1} 0\right\} \\
& \vdots \\
S_{k} & =\left\{0, T 0, T^{2} 0, T^{3} 0, \ldots, T^{t^{(k)}-1} 0\right\}
\end{aligned}
$$

After ordering each set $S_{k}(k \geq 1)$, we write $S_{k}=\left\{p_{0}^{(k)}, p_{1}^{(k)}, \ldots, p_{t^{(k)}-1}^{(k)}\right\}$ where $0=p_{0}^{(k)}<p_{1}^{(k)}<\cdots<p_{t^{(k)}-1}^{(k)}<1=p_{t^{(k)}}^{(k)}$.

For $0 \leq j<t^{(k)}$, each interval $\left[p_{j}^{(k)}, p_{j+1}^{(k)}\right)$ is called a $k$-canonical cylinder or just a canonical cylinder in $X$ and each point $p_{j}^{(k)}$ is called a $k$ canonical point. A finite or countable union of canonical cylinders is called a cylinder in $X$. Clearly each cylinder is a Borel subset of $X$.

A set of cylinders $\left\{C_{1}, C_{2}, \ldots, C_{n}\right\}$ is said to be iterated if there exists some $C_{j}(1 \leq j \leq n)$ such that the set of cylinders $\left\{C_{1}, C_{2}, \ldots, C_{n}\right\}$ is the same as the set of cylinders $\left\{C_{j}, T C_{j}, T^{2} C_{j}, \ldots, T^{n-1} C_{j}\right\}$.

Note that the cylinders belonging to an iterated set of cylinders have the same length.

$\S$ 2.2. Some basic results concerning the canonical cylinders. These facts either are standard or follow easily from standard facts about continued fractions. 
(i)

$$
\begin{gathered}
\left.\begin{array}{c}
T_{2}^{t_{2}^{(k)}} 0=\alpha_{k-1} \\
T_{1}^{t_{1}^{(k)}} 0=1-\alpha_{k}
\end{array}\right\} \quad \text { if } k \text { is odd } \\
\left.\begin{array}{c}
T_{1}^{t_{1}^{(k)}} 0=\alpha_{k} \\
T_{2}^{t_{2}^{(k)}} 0=1-\alpha_{k-1}
\end{array}\right\} \quad \text { if } k \text { is even }
\end{gathered}
$$

(ii) If $k$ is even, then $\left[0, T_{1}^{t_{1}^{(k)}} 0\right)$ has length $\alpha_{k}$ and $\left[T_{2}^{t_{2}^{(k)}} 0,1\right)$ has length $\alpha_{k-1}$. If $k$ is odd, then $\left[0, T_{2}^{t^{(k)}} 0\right)$ has length $\alpha_{k-1}$ and $\left[T_{1}^{t_{1}^{(k)}} 0,1\right)$ has length $\alpha_{k}$.

Note. If a $k$-canonical cylinder has length $\alpha_{k-1}$ we call it a long $k$ canonical cylinder, if it has length $\alpha_{k}$ we call it a short $k$-canonical cylinder.

(iii) If $k$ is even there are $t_{2}^{(k)}$ short $k$-canonical cylinders $T^{j}\left(\left[0, T^{t_{1}^{(k)}} 0\right)\right)$ for $0 \leq$ $j<t_{2}^{(k)}$ and $t_{1}^{(k)}$ long $k$-canonical cylinders $T^{j}\left(\left[T^{t_{2}^{(k)}} 0,1\right)\right)$ for $0 \leq j<t_{1}^{(k)}$. If $k$ is odd there are $t_{1}^{(k)}$ long $k$-canonical cylinders $T^{j}\left(\left[0, T_{2}^{t_{2}^{(k)}} 0\right)\right)$ for $0 \leq$ $j<t_{1}^{(k)}$ and $t_{2}^{(k)}$ short $k$-canonical cylinders $T^{j}\left(\left[T^{t_{1}^{(k)}} 0,1\right)\right)$ for $0 \leq j<t_{2}^{(k)}$.

(iv) A short $k$-canonical cylinder is equal to a long $(k+1)$-canonical cylinder and a long $k$-canonical cylinder is split into $\left(a_{k+1}+1\right)(k+1)$-canonical cylinders among which $a_{k+1}$ are long and one is short.

\section{$\S$ 2.3. Cylinder partition}

A cylinder partition of $X$ is a set $\mathcal{C}$ of pairwise disjoint cylinders s.t.

$$
\sum_{c \in \mathcal{C}} \mu(c)=1
$$

$\S$ 2.4. Canonical cylinder partition

We have seen that there are $t^{(k)} k$-canonical cylinders $\left[p_{j}^{(k)}, p_{j+1}^{(k)}\right)$ for $0 \leq j<t^{(k)}$. Since $0=p_{0}^{(k)}<p_{1}^{(k)}<\cdots<p_{t^{(k)}-1}^{(k)}<1=p_{t^{(k)}}^{(k)}$, the $k$-canonical cylinders are pairwise disjoint and the sum of their $\mu$-measures is 1 . So the $k$ canonical cylinders $(k \geq 1)$ form a cylinder partition. Such a cylinder partition is called the canonical cylinder partition of $X$.

\section{$\S 2.5$. Tower partition}

A tower partition is a cylinder partition $\mathcal{C}$ endowed with an equivalence relation in which each equivalence class consists of canonical cylinders having the same measure (or length). 
For any two canonical cylinders $c_{1}, c_{2}$ with $\mu\left(c_{1}\right)=\mu\left(c_{2}\right)$ there is a unique $t \in \mathbb{Z}$ with $T^{t}\left(c_{1}\right)=c_{2}$. We endow each equivalence class of a tower partition with the order inherited from the value $t$, i.e. $c_{1}<c_{2}$ if and only if $T^{t}\left(c_{1}\right)=c_{2}$ and $t>0$.

Each equivalence class $C$ of the tower partition $\mathcal{C}$ is called a tower or a column of $\mathcal{C}$. The number of elements in $C$ is called the height of the tower $C$.

By the support of $C$ we mean

$$
\operatorname{Supp}(C)=\cup_{c \in C} c
$$

$\S$ 2.6. Canonical tower partition $\mathcal{P}_{k}(k \geq 1)$

Each canonical cylinder partition $\left\{\left[p_{j}^{(k)}, p_{j+1}^{(k)}\right): 0 \leq j<t^{(k)}\right\}(k \geq 1)$ consists of two equivalence classes, one equivalence class is of height $t_{1}^{(k)}$ and it consists of $t_{1}^{(k)}$ iterated canonical cylinders all having the same $\mu$-measure $\alpha_{k-1}$ and the other equivalence class is of height $t_{2}^{(k)}$ and it consists of $t_{2}^{(k)}$ iterated canonical cylinders all having the same $\mu$-measure $\alpha_{k}$ and endowed with increasing order up the towers as induced by the action of $T$. This tower partition is called the canonical tower partition and we denote it by $\mathcal{P}_{k}$.

\section{$\S$ 2.7. Common extension}

Let $C=\left\{c_{0}, T^{n_{1}} c_{0}, T^{n_{2}} c_{0}, \ldots, T^{n_{k}} c_{0}\right\}$ be an ordered column of a tower partition $\mathcal{C}$ and $c_{0}^{\prime} \subseteq c_{0}$ be a canonical cylinder. Then we call the ordered set $\left\{c_{0}^{\prime}, T^{n_{1}} c_{0}^{\prime}, T^{n_{2}} c_{0}^{\prime}, \ldots, T^{n_{k}} c_{0}^{\prime}\right\}$ a common extension of $C$ by $c_{0}^{\prime}$.

A tower partition $\hat{\mathcal{C}}$ is said to be a common extension of a tower partition $\mathcal{C}$ if each column of $\hat{\mathcal{C}}$ is a common extension of a column of $\mathcal{C}$.

$\S$ 2.8. Tower refinement

Let $\mathcal{C}$ be a tower partition and $\hat{\mathcal{C}}$ be a common extension of $\mathcal{C}$. Then a tower partition $\mathcal{C}^{\prime}$ is a refinement of $\mathcal{C}$ if each column of $\mathcal{C}^{\prime}$ is a union of columns of $\hat{\mathcal{C}}$ and the order on $\mathcal{C}^{\prime}$ extends the order on $\hat{\mathcal{C}}$.

Note that if $\mathcal{C}^{\prime}$ is a tower refinement of $\mathcal{C}$, then each $c^{\prime} \in \mathcal{C}^{\prime}$ is contained in a unique $c \in \mathcal{C}$, yielding a map $\pi: \mathcal{C}^{\prime} \rightarrow \mathcal{C}$ which preserves the measure $\mu$. Note also that the set $\hat{\mathcal{C}}$ is identical with the set $\mathcal{C}^{\prime}$, but that its equivalence relation is finer (equivalence classes of the former are subsets of the equivalence classes of the latter). For any $c^{\prime} \in \mathcal{C}^{\prime}$ by $\left[c^{\prime}\right]$ we denote the equivalence class in $\mathcal{C}^{\prime}$ containing $c^{\prime}$ and by $\left[\left[c^{\prime}\right]\right]$ we denote the equivalence class in $\hat{\mathcal{C}}$ containing $c^{\prime}$. 
§ 2.9. $(1-\epsilon) \mathcal{P}_{k}$-invariance

Let $0<\epsilon<1$ and $k \geq 1$. Then a tower partition $\mathcal{C}$ of $X$ is called $(1-\epsilon) \mathcal{P}_{k^{-}}$ invariant if there exists a common extension $\mathcal{P}_{k}^{\prime}$ of $\mathcal{P}_{k}$ such that

$$
\sum_{D} \sum_{c \in D} \mu(c) \geq 1-\epsilon
$$

where the sum $\sum_{D}$ is taken over all columns $D$ in $\mathcal{P}_{k}^{\prime}$ such that all cylinders $c \in D$ are $\mathcal{C}$-equivalent.

The set of all such columns $D$ in $\mathcal{P}_{k}^{\prime}$ in the above inequality is called the major portion of $\mathcal{C}$ and is denoted by $\operatorname{Major}(\mathcal{C})$.

Remark. This definition is more generally defined in terms of measured Bratteli diagram in [HKR].

The following lemma is useful in proving the Proposition 4.2.

For $\gamma \in(0,1]$, we define inductively

$$
\begin{aligned}
\gamma_{1} & =\gamma \\
n_{k} & =\min \left\{n \geq 1: 2 \alpha_{n-1} \leq \gamma_{k}\right\} \quad(k \geq 1) \\
\gamma_{k+1} & =\gamma_{k}-\alpha_{n_{k}-1} \quad(k \geq 1) .
\end{aligned}
$$

Then $1 \leq n_{1} \leq n_{2} \leq \cdots$ and $\left\{\gamma_{k}\right\}$ is a monotone decreasing sequence of real numbers and bounded below by 0 , and hence $\gamma_{k} \rightarrow 0$ as $k \rightarrow \infty$, and so

$$
\gamma=\sum_{k=1}^{\infty} \alpha_{n_{k}-1} .
$$

We say that $n=\left(n_{1}, n_{2}, \ldots\right)$ is the $\gamma$-universal sequence.

Lemma 2.10. Let $0<\gamma \leq 1$. Then any subinterval of the unit interval of length $\gamma$ can be written as a disjoint union of long $n_{k}$-canonical cylinders, one cylinder for each $k \geq 1$, where $n=\left(n_{1}, n_{2}, \ldots\right)$ is the $\gamma$-universal sequence.

Proof. As $2 \alpha_{n_{1}-1} \leq \gamma_{1}$, the given interval must contain a long $n_{1^{-}}$ canonical cylinder. Remove it, leaving two intervals each with at least one $n_{1}$-canonical end point. One of these intervals has length at least $\frac{\gamma_{2}}{2}$, and thus contains at one end a long $n_{2}$-canonical cylinder. Remove it and proceed by induction. 
Proposition 2.11. Let $\mathcal{C}$ be a tower partition of $X$ and let $0<\epsilon<1$. Then for all sufficiently large $k$ there exists a $(1-\epsilon) \mathcal{P}_{k}$-invariant tower refinement $\mathcal{C}^{\prime}$ of $\mathcal{C}$.

Proof. Let $C_{1}, C_{2}, C_{3}, \cdots$ be the columns in $\mathcal{C}$. Choose $k_{0}$ such that

$$
\sum_{i=1}^{k_{0}} \mu\left(\operatorname{Supp}\left(C_{i}\right)\right) \geq 1-\epsilon .
$$

Let $k \geq k_{0}$ be arbitrary. We want to construct a tower refinement $\mathcal{C}^{\prime}$ of $\mathcal{C}$ and a common extension $\mathcal{P}_{k}^{\prime}$ of $\mathcal{P}_{k}$ such that there exists many columns $P^{\prime}$ of $\mathcal{P}_{k}^{\prime}$ with $P^{\prime} \subseteq$ some $\mathcal{C}^{\prime}$-column and the total $\mu$-measure of the supports of these $P^{\prime} \geq 1-\epsilon$. For any $l \geq k$ we can construct a tower refinement $\mathcal{C}_{l}^{\prime}$ of $\mathcal{C}$ as follows:

Cylinders in each $C_{i}$ are $j_{i}$-canonical cylinders for some $j_{i}$. If $j_{i} \leq l$ take all possible common extensions of $C_{i}$ so that the cylinders in each common extension become $l$-canonical cylinders. If $j_{i}>l$ leave it alone. Let $C_{l}$ be the union of all the common extensions which contain only the long $l$-canonical cylinders and $C_{l}^{\prime}$ be the union of all the common extensions which contain only the short $l$-canonical cylinders, both endowed with an order which extends the order on $\mathcal{C}$. These two columns and the remaining columns which were left alone form the tower refinement $\mathcal{C}_{l}^{\prime}$ of $\mathcal{C}$.

Let $\delta_{l}$ be the sum of the $\mu$-measures of the cylinders in $\mathcal{C}_{l}^{\prime}$ which do not belong to the column $C_{l}$ or $C_{l}^{\prime}$. Choose $l$ so large that

$$
\delta_{l}<\frac{\epsilon}{t_{1}^{(k)}} .
$$

Let $\mathcal{P}_{k}^{\prime}$ be the common extension of $\mathcal{P}_{k}$ such that columns in $\mathcal{P}_{k}^{\prime}$ are formed by $l$-canonical cylinders. Mark all $l$-canonical cylinders in $\mathcal{P}_{k}^{\prime}$ which do not belong to the column $C_{l}$ or $C_{l}^{\prime}$ in $\mathcal{C}_{l}^{\prime}$. Clearly such $l$-canonical cylinders not belonging to the column $C_{l}$ or $C_{l}^{\prime}$ has total $\mu$-measure $\delta_{l}$. Let $\mathcal{F}_{P}$ be the collection of all the columns in $\mathcal{P}_{k}^{\prime}$ which contain at least one of these marked cylinders. Then

$$
\sum_{C_{P} \in \mathcal{F}_{P}} \mu\left(\operatorname{Supp}\left(C_{P}\right)\right) \leq t_{1}^{(k)} \delta_{l}<t_{1}^{(k)} \frac{\epsilon}{t_{1}^{(k)}}=\epsilon .
$$

Therefore, the cylinders in all other columns $D$ in $\mathcal{P}_{k}^{\prime}$ are $\mathcal{C}_{l}^{\prime}$-equivalent and

$$
\sum_{D} \sum_{c \in D} \mu(c) \geq 1-\epsilon
$$


This $\mathcal{C}_{l}^{\prime}$ is a $(1-\epsilon) \mathcal{P}_{k}$-invariant tower refinement $\mathcal{C}^{\prime}$ of $\mathcal{C}$. Since $k \geq k_{0}$ is arbitrary, so the proposition is true for all sufficiently large $k$.

Remark. This proposition is more generally proved in terms of ergodic measured Bratteli diagram in [HKR].

\section{$\S 3 . \quad$ Binary Odometer}

In this section we discuss the basic definitions, lemmas and proposition concerning the binary odometer. For this section we are indebted to Hamachi and Keane $[\mathrm{HK}]$.

$\S$ 3.1. Odometer, cylinder and dyadic interval

Let $Y=\{0,1\}^{\mathbb{N}}$. Then $Y$ is a product topological space arising from the discrete topology on each of its coordinate space $\{0,1\} . Y$ is metrizable. The metric

$$
d\left(y, y^{\prime}\right)=2^{-l} \text { where } l=\min \left\{|i|: y_{i} \neq y_{i}^{\prime}\right\}
$$

generates the product topology on $Y$. The open balls in the product topology are

$$
B\left(y, 2^{-l}\right)=\left\{y^{\prime}=\left(y_{n}^{\prime}\right) \in Y: y_{i}=y_{i}^{\prime}, 0 \leq i \leq l\right\}
$$

Let

$$
W=\{0,1\}^{*}=\cup_{l \in \mathbb{N}}\{0,1\}^{l}
$$

denotes the set of all binary words. If $d \in W$ then $|d|$ denotes its length; the empty word $\emptyset$ has length 0 . Each word $d \in W$ gives rise to the subset

$$
\{y \in Y \mid y \text { begins with } d\}
$$

of $Y$, which we call a cylinder in $Y$ and also denote by the same letter $d$. If $y \in d$ then by (2) we have

$$
d=B\left(y, 2^{-|d|}\right)
$$

The clopen sets of $Y$ are the finite unions of cylinders. Let us now define,

$\mathfrak{B}:=$ the Borel $\sigma$-algebra of $Y$ generated by the cylinders in $Y$,

$$
\nu:=\left(\frac{1}{2}, \frac{1}{2}\right)^{\mathbb{N}}=\text { the product measure on }(Y, \mathfrak{B}) \text {. }
$$


Under the coordinatewise addition modulo 2 with right carry $1, Y$ is a compact topological group and $\nu$ is the normalized Haar measure on $Y$. Moreover, since the subgroup generated by 1 is dense, $Y$ is monothetic.

The invertible ergodic measure preserving transformation $S: Y \rightarrow Y$ defined by $S y=y+1$, where $1=(1,0,0, \cdots)$ is called the binary odometer or the binary adding machine on $(Y, \mathfrak{B}, \nu)$. Note that $S$ is a homeomorphism of the compact group $Y$, since it is the translation by the element $1=(1,0,0, \cdots)$ of this group.

A dyadic interval of level $k$ or a $k$-dyadic interval is defined as an interval

$$
\left[\frac{l}{2^{k}}, \frac{l+1}{2^{k}}\right)
$$

with $0 \leq l<2^{k}$; these are just cylinders $d \in W$ with $|d|=k$ in another guise. By a canonical cylinder in $Y$ we just mean a dyadic interval in $Y$ of some level $k \geq 0$.

$\S$ 3.2. Cylinder partition, Tower partition, Support, Strict extension, Tower refinement

These definitions for the binary odometer are exactly same as it is done for the irrational rotation of the circle. But in this case a tower partition is denoted by $\mathcal{D}$ and a cylinder by $d$.

§3.3. $(1-\epsilon)$-cyclic

A tower partition $\mathcal{D}$ of $Y$ is called $(1-\epsilon)$-cyclic if it has a column $D$ such that

$$
\nu(\operatorname{Supp}(D)) \geq 1-\epsilon
$$

The following simple lemma about base 2 expansion is useful in proving the Proposition 4.1.

For $\delta \in(0,1]$, we define inductively

$$
\begin{aligned}
\delta_{1} & =\delta \\
n_{k} & =\min \left\{n \geq 1: \frac{2}{2^{n}} \leq \delta_{k}\right\} \quad(k \geq 1) \\
\delta_{k+1} & =\delta_{k}-\frac{1}{2^{n_{k}}} \quad(k \geq 1) .
\end{aligned}
$$

Then $1 \leq n_{1} \leq n_{2} \leq \cdots$ and $\delta=\sum_{k=1}^{\infty} \frac{1}{2^{n_{k}}}$. We say that $n=\left(n_{1}, n_{2}, \cdots\right)$ is the $\delta$-universal sequence. 
Lemma 3.4. Let $0<\delta \leq 1$. Then any subinterval of the unit interval of length $\delta$ can be written as a disjoint union of $n_{k}$-dyadic intervals, one interval for each $k \geq 1$, where $n=\left(n_{1}, n_{2}, \cdots\right)$ is the $\delta$-universal sequence.

Proposition 3.5. Let $\mathcal{D}$ be a tower partition of $Y$ and let $0<\epsilon<1$. Then there exists a $(1-\epsilon)$-cyclic tower refinement $\mathcal{D}^{\prime}$ of $\mathcal{D}$.

Proof. Select a finite set of columns from $\mathcal{D}$ with total $\nu$-measure of their supports is at least $(1-\epsilon)$. Let $l$ be the minimum of the $\nu$-measures of the canonical cylinders belonging to the selected columns. Take all possible common extensions of the selected columns so that all the cylinders in the common extensions have the same $\nu$-measure $l$. Let $D^{\prime}$ be the union of these common extensions endowed with an order which extends the order on $\mathcal{D}$.

Let us now form $\mathcal{D}^{\prime}$ by taking $D^{\prime}$ as a column and the remaining columns of $\mathcal{D}^{\prime}$ are the remaining columns of $\mathcal{D}$ which were not selected before. Then $\mathcal{D}^{\prime}$ is a $(1-\epsilon)$-cyclic tower refinement of $\mathcal{D}$.

\section{$\S 4 . \quad$ Tower Maps}

The concept of tower maps is central to our construction.

Let $\mathcal{C}$ and $\mathcal{D}$ be tower partitions of $X$ and $Y$. A tower map is a mapping

$$
\phi: \mathcal{C} \rightarrow \mathcal{D}
$$

taking each $\mathcal{C}$-equivalence class bijectively to a $\mathcal{D}$-equivalence class, and taking $\mu$ to $\nu$ :

$$
\nu(d)=\sum_{\{c \in \mathcal{C}: \phi(c)=d\}} \mu(c) \quad(d \in \mathcal{D}) .
$$

Tower maps in the other direction are defined in an analogous manner.

The following two propositions we need in constructing the tower refinements. In a similar way as Proposition 2 in [HK], these two propositions will be obtained and proven.

Proposition 4.1. Let $\phi: \mathcal{C} \rightarrow \mathcal{D}$ be a tower map, and let $\mathcal{C}^{\prime}$ be a tower refinement of $\mathcal{C}$. Then there exists a tower refinement $\mathcal{D}^{\prime}$ of $\mathcal{D}$ and a tower map $\psi: \mathcal{D}^{\prime} \rightarrow \mathcal{C}^{\prime}$ such that the diagram

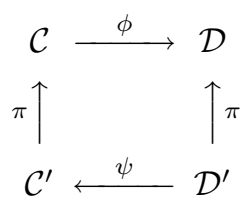


commutes, i.e. $\phi \circ \pi \circ \psi=\pi$, and such that $\psi$ is also a tower map from $\hat{\mathcal{D}}$ to $\hat{\mathcal{C}}$, where $\hat{\mathcal{C}}(\hat{\mathcal{D}})$ are the tower extensions corresponding to $\mathcal{C}^{\prime}\left(\mathcal{D}^{\prime}\right)$.

Proof. It will be convenient to identify cylinders in $Y$ with dyadic intervals. First choose from each $\mathcal{D}$-equivalence class one representative $d$ and identify it with its dyadic interval of length $\frac{1}{2^{|d|}}=\nu(d)$. Then

$$
\sum_{\left\{c^{\prime} \in \mathcal{C}^{\prime}: \phi\left(\pi\left(c^{\prime}\right)\right)=d\right\}} \mu\left(c^{\prime}\right)=\nu(d),
$$

so that we can choose for each such $c^{\prime}$ a subinterval $I\left(d, c^{\prime}\right)$ of $d$ such that $\nu\left(I\left(d, c^{\prime}\right)\right)=\mu\left(c^{\prime}\right)$ and which together cover $d$. We make and fix this choice in some manner. Now by Lemma 3.4, to each such subinterval $I\left(d, c^{\prime}\right)$ of $d$ there exists a sequence $\left\{I_{n_{k}}\left(d, c^{\prime}\right)\right\}_{k \geq 1}$ of disjoint $n_{k}$-dyadic intervals such that

$$
I_{n_{k}}\left(d, c^{\prime}\right) \subset I\left(d, c^{\prime}\right) \quad \text { and } \quad \cup_{k \geq 1} I_{n_{k}}\left(d, c^{\prime}\right)=I\left(d, c^{\prime}\right)
$$

where $n=\left(n_{1}, n_{2}, n_{3}, \cdots\right)$ is the $\delta$-universal sequence, $\delta$ being the length of $I\left(d, c^{\prime}\right)$. Note that by using the lemma we are guaranteed the same lengths $\frac{1}{2^{n_{k}}}$ of $I_{n_{k}}\left(d, c^{\prime}\right)$ for all $c^{\prime}$ having the same length.

For each $k \geq 1$ and each equivalence class $\left[\left[c^{\prime}\right]\right]$ of $\hat{\mathcal{C}}$ take the set of cylinders

$$
\left\{I_{n_{k}}\left(\tilde{d}, \tilde{c}^{\prime}\right): \phi\left(\pi\left(\tilde{c}^{\prime}\right)\right)=\tilde{d}, \tilde{c}^{\prime} \sim c^{\prime} \text { and } \tilde{c}^{\prime} \in\left[\left[c^{\prime}\right]\right]\right\},
$$

in which we preserve the same order inherited from $\mathcal{D}$. These sets define a tower partition of $Y$ which we denote by $\hat{\mathcal{D}}$. Now we take for each $k \geq 1$ and each equivalence class $\left[c^{\prime}\right]$ of $\mathcal{C}^{\prime}$ the set of cylinders

$$
\left\{I_{n_{k}}\left(\tilde{d}, \tilde{c}^{\prime}\right): \phi\left(\pi\left(\tilde{c}^{\prime}\right)\right)=\tilde{d}, \tilde{c}^{\prime} \sim c^{\prime} \text { and } \tilde{c}^{\prime} \in\left[c^{\prime}\right]\right\}
$$

endowed with an order which extends the order on $\mathcal{D}$. These sets define a tower partition of $Y$ which we denote by $\mathcal{D}^{\prime}$. Clearly $\hat{\mathcal{D}}$ is a tower extension of $\mathcal{D}$, where $\mathcal{D}^{\prime}$ is a tower refinement of $\mathcal{D}$. Put

$$
\psi\left(I_{n_{k}}\left(d, c^{\prime}\right)\right)=c^{\prime}, \quad I_{n_{k}}\left(d, c^{\prime}\right) \in \mathcal{D}^{\prime} .
$$

Then $\psi$ is a tower map from $\mathcal{D}^{\prime}$ to $\mathcal{C}^{\prime}$, and a tower map from $\hat{\mathcal{D}}$ to $\hat{\mathcal{C}}$. The commutativity $\phi \circ \pi \circ \psi=\pi$ follows from

$$
(\phi \circ \pi \circ \psi)\left(I_{n_{k}}\left(d, c^{\prime}\right)\right)=\phi\left(\pi\left(c^{\prime}\right)\right)=d=\pi\left(I_{n_{k}}\left(d, c^{\prime}\right)\right)
$$

and the proof is finished. 
Proposition 4.2. Let $\psi: \mathcal{D} \rightarrow \mathcal{C}$ be a tower map, and let $\mathcal{D}^{\prime}$ be a tower refinement of $\mathcal{D}$. Then there exists a tower refinement $\mathcal{C}^{\prime}$ of $\mathcal{C}$ and a tower map $\phi: \mathcal{C}^{\prime} \rightarrow \mathcal{D}^{\prime}$ such that the diagram

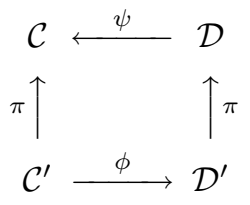

commutes, i.e. $\psi \circ \pi \circ \phi=\pi$, and such that $\phi$ is also a tower map from $\hat{\mathcal{C}}$ to $\hat{\mathcal{D}}$, where $\hat{\mathcal{C}}(\hat{\mathcal{D}})$ are the tower extensions corresponding to $\mathcal{C}^{\prime}\left(\mathcal{D}^{\prime}\right)$.

Proof. First choose from each $\mathcal{C}$-equivalence class one representative $c$. Then

$$
\sum_{\left\{d^{\prime} \in \mathcal{D}^{\prime}: \psi\left(\pi\left(d^{\prime}\right)\right)=c\right\}} \nu\left(d^{\prime}\right)=\mu(c),
$$

so that we can choose for each such $d^{\prime}$ a subinterval $I\left(c, d^{\prime}\right)$ of $c$ of length $\frac{1}{2^{\left|d^{\prime}\right|}}$ which together cover $c$. We make and fix this choice in some manner. Now by Lemma 2.10, to each such subinterval $I\left(c, d^{\prime}\right)$ of $c$ there exists a sequence $\left\{I_{n_{k}}\left(c, d^{\prime}\right)\right\}_{k \geq 1}$ of disjoint long $n_{k}$-canonical cylinders in $X$ such that

$$
I_{n_{k}}\left(c, d^{\prime}\right) \subset I\left(c, d^{\prime}\right) \quad \text { and } \quad \cup_{k \geq 1} I_{n_{k}}\left(c, d^{\prime}\right)=I\left(c, d^{\prime}\right)
$$

where $n=\left(n_{1}, n_{2}, n_{3}, \cdots\right)$ is the $\gamma$-universal sequence, $\gamma$ being the length of $I\left(c, d^{\prime}\right)$. Note that by using the lemma we are guaranteed the same lengths $\alpha_{n_{k}-1}$ of $I_{n_{k}}\left(c, d^{\prime}\right)$ for all $d^{\prime}$ having the same length.

For each $k \geq 1$ and each equivalence class $\left[\left[d^{\prime}\right]\right]$ of $\hat{\mathcal{D}}$ take the set of cylinders

$$
\left\{I_{n_{k}}\left(\tilde{c}, \tilde{d}^{\prime}\right): \psi\left(\pi\left(\tilde{d}^{\prime}\right)\right)=\tilde{c}, \tilde{d}^{\prime} \sim d^{\prime} \text { and } \tilde{d}^{\prime} \in\left[\left[d^{\prime}\right]\right]\right\},
$$

in which we preserve the same order inherited from $\mathcal{C}$. These sets define a tower partition of $X$ which we denote by $\hat{\mathcal{C}}$. Now we take for each $k \geq 1$ and each equivalence class $\left[d^{\prime}\right]$ of $\mathcal{D}^{\prime}$ the set of cylinders

$$
\left\{I_{n_{k}}\left(\tilde{c}, \tilde{d}^{\prime}\right): \psi\left(\pi\left(\tilde{d}^{\prime}\right)\right)=\tilde{c}, \tilde{d}^{\prime} \sim d^{\prime} \text { and } \tilde{d}^{\prime} \in\left[d^{\prime}\right]\right\}
$$

endowed with an order which extends the order on $\mathcal{C}$. These sets define a tower partition of $X$ which we denote by $\mathcal{C}^{\prime}$. Clearly $\hat{\mathcal{C}}$ is a tower extension of $\mathcal{C}$, where $\mathcal{C}^{\prime}$ is a tower refinement of $\mathcal{C}$. Put

$$
\phi\left(I_{n_{k}}\left(c, d^{\prime}\right)\right)=d^{\prime}, \quad I_{n_{k}}\left(c, d^{\prime}\right) \in \mathcal{C}^{\prime} .
$$


Then $\phi$ is a tower map from $\mathcal{C}^{\prime}$ to $\mathcal{D}^{\prime}$, and a tower map from $\hat{\mathcal{C}}$ to $\hat{\mathcal{D}}$. The commutativity $\psi \circ \pi \circ \phi=\pi$ follows from

$$
(\psi \circ \pi \circ \phi)\left(I_{n_{k}}\left(c, d^{\prime}\right)\right)=\psi\left(\pi\left(d^{\prime}\right)\right)=c=\pi\left(I_{n_{k}}\left(c, d^{\prime}\right)\right)
$$

and the proof is finished.

\section{$\S 5$. The Finitary Construction}

Two points are in the same column, by this it is meant, the canonical cylinders containing them are in the same column.

The following proposition will play an important role in the finitary construction.

Proposition 5.1. For a sequence $\mathcal{C}^{n}(n \geq 0)$ of tower partitions of $X$ with $\mathcal{C}^{n+1}$ is a tower refinement of $\mathcal{C}^{n}$, if $x$ and $\tilde{x}$ be two points in $X$ and they are in the same column of $\mathcal{C}^{n}$ for all sufficiently large $n$, then they are in the same orbit under the irrational rotation $T$.

Proof. Let $x \in c_{n}(x) \in \mathcal{C}^{n}$ and $\tilde{x} \in c_{n}(\tilde{x}) \in \mathcal{C}^{n}$.

By the given condition $\exists n_{0} \in \mathbb{N}$ s.t. $\forall n \geq n_{0}$

$$
c_{n}(x) \sim c_{n}(\tilde{x}),
$$

and so $\exists$ an integer $k$ s.t.

$$
c_{n}(\tilde{x})=T^{k}\left(c_{n}(x)\right) \quad \forall n \geq n_{0} .
$$

Since $c_{0}(x) \stackrel{\pi}{\longleftarrow} c_{1}(x) \stackrel{\pi}{\leftarrow} c_{2}(x) \leftarrow \cdots$ is a chain,

$$
\therefore \lim _{n \rightarrow \infty} c_{n}(x)=\bigcap_{n \geq 0} c_{n}(x)=\{x\} .
$$

Similarly,

$$
\lim _{n \rightarrow \infty} c_{n}(\tilde{x})=\bigcap_{n \geq 0} c_{n}(\tilde{x})=\{\tilde{x}\} .
$$

Since $T$ is continuous from (3) we have,

$$
\begin{gathered}
\{\tilde{x}\}=\lim _{n \rightarrow \infty} c_{n}(\tilde{x})=\lim _{n \rightarrow \infty} T^{k}\left(c_{n}(x)\right)=T^{k}\left(\lim _{n \rightarrow \infty} c_{n}(x)\right)=T^{k}(\{x\}) . \\
\therefore \quad \tilde{x}=T^{k}(x) .
\end{gathered}
$$

Hence $x$ and $\tilde{x}$ are in the same orbit under $T$. 
Remark. Analogous proposition is also true for the binary odometer.

Proof of Main Theorem. Choose a sequence $\left\{\epsilon_{n}\right\}$ with $0<\epsilon_{n}<1$ and

$$
\sum_{n=1}^{\infty} \epsilon_{n}<\infty .
$$

Using Propositions 2.11, 3.5, 4.1 and 4.2 inductively, produce the diagram

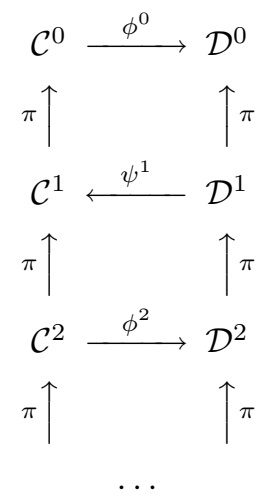

in which $\mathcal{C}^{0}=\{\emptyset\}, \mathcal{D}^{0}=\{\emptyset\}, \mathcal{C}^{2 n+1}$ is $\left(1-\epsilon_{2 n+1}\right) \mathcal{P}_{m_{2 n+1}}$-invariant, where the sequence $\left\{m_{n}\right\}$ is increasing $(n \geq 0) ; \mathcal{D}^{2 n}$ is $\left(1-\epsilon_{2 n}\right)$-cyclic $(n \geq 1)$ and the maps "commute".

Let

$$
N_{k}=X-\bigcup_{c \in \mathcal{C}^{k}} c \quad(k \geq 0) .
$$

Since $\sum_{c \in \mathcal{C}^{k}} \mu(c)=1$, so $\mu\left(N_{k}\right)=0$. Let $N=\bigcup_{k \geq 0} N_{k}$. Then $\mu(N)=0$.

By the definition of cylinder partition every $x \in(X-N)$ belongs to exactly one cylinder $c \in \mathcal{C}^{n}$ for each $n \geq 0$. Let us call this cylinder $c_{n}(x)$. So $x \in c_{n}(x) \in \mathcal{C}^{n} \forall n \geq 0$. Similarly $\exists$ a $\nu$-null subset $N^{\prime}$ of $Y$ such that every $y \in\left(Y-N^{\prime}\right)$ belongs to a cylinder $d_{n}(y) \in \mathcal{D}^{n}$ for each $n \geq 0$.

Moreover, if $d_{0} \stackrel{\pi}{\leftarrow} d_{1} \stackrel{\pi}{\leftarrow} d_{2} \leftarrow \cdots$ is a chain, then

$$
\bigcap_{n \geq 0} d_{n}
$$

is a singleton in $Y$. Therefore, we can define a function $\Phi:(X-N) \rightarrow\left(Y-N^{\prime}\right)$ given by $\Phi(x)=y$ such that for each $n \geq 0$,

$$
\phi^{2 n}\left(c_{2 n}(x)\right)=d_{2 n}(y) .
$$


Analogously (or by "commutativity") there exists a function $\Psi:\left(Y-N^{\prime}\right) \rightarrow$ $(X-N)$ given by $\Psi(y)=x$ such that for each $n \geq 0$,

$$
\psi^{2 n+1}\left(d_{2 n+1}(y)\right)=c_{2 n+1}(x),
$$

proving that $\Phi$ and $\Psi$ are inverses of each other.

Since for any $d \in \mathcal{D}^{2 n}(n \geq 0)$,

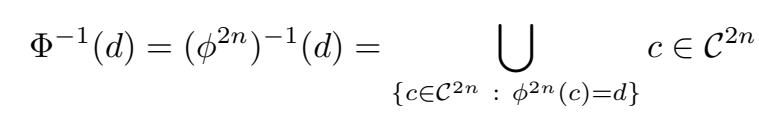

and $\phi^{2 n}$ being a tower map,

$$
\begin{aligned}
& \mu\left(\Phi^{-1}(d)\right)=\mu\left(\left(\phi^{2 n}\right)^{-1}(d)\right)
\end{aligned}
$$

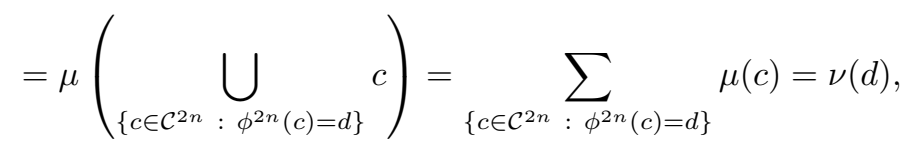

$\Phi$ is measurable and measure preserving.

Again each canonical cylinder is an open set up to a set of measure zero. Therefore, $\Phi$ is continuous after restriction to sets of measure one and so $\Phi$ is a finitary homomorphism. Similarly $\Psi:\left(Y-N^{\prime}\right) \rightarrow(X-N)$ is a finitary homomorphism and hence $\Phi$ is a finitary isomorphism.

Finally to show $S$ and $T$ are orbit equivalent under the mapping $\Phi$, let $x$ and $\tilde{x}$ be two points of $X$ belonging to the same orbit under the irrational circle rotation $T$. Let us take $\tilde{x}=T^{k} x$ for some $k \in \mathbb{N}$. Then by $\left(1-\epsilon_{2 n+1}\right) \mathcal{P}_{m_{2 n+1}}$ invariance and the Borel-Cantelli lemma $\mu$-almost every such $x$ belongs to a canonical cylinder $c_{2 n+1}(x)$ contained in a column in the major portion of $\mathcal{C}^{2 n+1}$ for all sufficiently large $n$.

Let for $n \geq 0, E_{2 n+1}$ be the union of all $k$ canonical cylinders taken from the top and also from the bottom of each columns in the major portion of $\mathcal{C}^{2 n+1}$. Then

$$
\mu\left(E_{2 n+1}\right) \leq 2 k\left(\alpha_{m_{2 n+1}-1}+\alpha_{m_{2 n+1}}\right) \leq 4 k \alpha_{m_{2 n+1}-1} \leq 4 k \alpha_{2 n} \leq 4 k \frac{1}{2^{2 n}},
$$

and therefore

$$
\sum_{n=0}^{\infty} \mu\left(E_{2 n+1}\right)<\infty .
$$

We can apply the Borel-Cantelli lemma again to say that for $\mu$-almost every $x$ and for all sufficiently large $n, x$ is not only in a canonical cylinder 
contained in a column in the major portion of $\mathcal{C}^{2 n+1}$ but also in

$$
\bigcup_{\substack{c \in D \\ D \in \operatorname{Major}\left(\mathcal{C}^{2 n+1}\right)}} c-E_{2 n+1} .
$$

This means that $\tilde{x}=T^{k} x$ and $x$ are in the same common extension of $\mathcal{P}_{m_{2 n+1}}$ for all sufficiently large $n$. In other words $x$ and $\tilde{x}$ are in the same column of $\mathcal{C}^{2 n+1}$ for all sufficiently large $n$, that is the canonical cylinders $c_{2 n+1}(x)$ and $c_{2 n+1}(\tilde{x})$ are in the same column of $\mathcal{C}^{2 n+1}$ for all sufficiently large $n$.

By the definition of Tower maps, $\phi^{2 n+2}$ takes a $\mathcal{C}^{2 n+2}$-column to a $\mathcal{D}^{2 n+2}$ column. Hence the corresponding canonical cylinders $d_{2 n+2}(\Phi(x))=\phi^{2 n+2}$ $\left(c_{2 n+2}(x)\right)$ and $d_{2 n+2}(\Phi(\tilde{x}))=\phi^{2 n+2}\left(c_{2 n+2}(\tilde{x})\right)$ are in the same column of $\mathcal{D}^{2 n+2}$ for all sufficiently large $n$. In other words, $y=\Phi(x)$ and $\tilde{y}=\Phi(\tilde{x})$ are in the same column of $\mathcal{D}^{n}$ for all sufficiently large $n$. Hence by the remark of Proposition 5.1, we have $y=\Phi(x)$ and $\tilde{y}=\Phi(\tilde{x})$ are in the same orbit under the binary odometer $S$.

Now let $y$ and $\tilde{y}$ be two points of $Y$ belonging to the same orbit under the binary odometer $S$ and suppose that this is not the orbit of 1 . Then it is well known that $y$ and $\tilde{y}$ agree in all but a finite number of coordinates. By $\left(1-\epsilon_{2 n}\right)$ cyclicity and the Borel-Cantelli lemma $\nu$-almost every such $y$ and $\tilde{y}$ belong to the cylinders in the "large" equivalence class of $\mathcal{D}^{2 n}$ for all sufficiently large $n$, that is the canonical cylinders $d_{2 n}(y)$ and $d_{2 n}(\tilde{y})$ are in the same column of $\mathcal{D}^{2 n}$ for all sufficiently large $n$.

By the definition of tower maps, $\psi^{2 n+1}$ takes a $\mathcal{D}^{2 n+1}$-column to a $\mathcal{C}^{2 n+1}$ column. Hence the corresponding canonical cylinders $c_{2 n+1}(\Psi(y))=\psi^{2 n+1}$ $\left(d_{2 n+1}(y)\right)$ and $c_{2 n+1}(\Psi(\tilde{y}))=\psi^{2 n+1}\left(d_{2 n+1}(\tilde{y})\right)$ are in the same column of $\mathcal{C}^{2 n+1}$ for all sufficiently large $n$. In other words, $x=\Psi(y)$ and $\tilde{x}=\Psi(\tilde{y})$ are in the same column of $\mathcal{C}^{n}$ for all sufficiently large $n$. Hence by Proposition 5.1, we have $x=\Psi(y)$ and $\tilde{x}=\Psi(\tilde{y})$ are in the same orbit under the irrational circle rotation $T$.

Hence $S$ and $T$ are orbit equivalent under the mapping $\Phi$.

Thus we have constructed an invertible measure preserving mapping $\Phi$ from a subset of $X$ of measure one to a subset of $Y$ of full measure such that $\Phi\left(\operatorname{Orbit}_{T}(x)\right)=\operatorname{Orbit}_{S}(\Phi(x))$ for $\mu$ a.e. $x \in X$ and both $\Phi$ and its inverse are continuous after restriction to sets of measure one. This proves the theorem. 


\section{References}

[D] H. A. Dye, On groups of measure preserving transformation. I, Amer. J. Math. 81 (1959), 119-159.

[HK] T. Hamachi and M. S. Keane, Finitary orbit equivalence of odometers, Bull. London Math. Soc. 38 (2006), no. 3, 450-458.

[HKR] T. Hamachi, M. S. Keane and M. K. Roychowdhury, Finitary orbit equivalence and measured Bratteli diagrams, to appear Colloquium Mathematicum.

[HO] T. Hamachi and M. Osikawa, Ergodic groups of automorphisms and Krieger's theorems, Seminar on Math. Keio Univ., 3 (1981).

[KS1] M. Keane and M. Smorodinsky, A class of finitary codes, Israel J. Math. 26 (1977), no. $3-4,352-371$.

[KS2] , Bernoulli schemes of the same entropy are finitarily isomorphic, Ann. of Math. (2) 109 (1979), no. 2, 397-406.

[R] M. K. Roychowdhury, $\left\{m_{n}\right\}$-odometer and the binary odometer are finitarily orbit equivalent, Contemporary Mathematics, AMS, Edited by I. Assani, UNC Chapel Hill, 430 (2007), 123-134. 